\title{
Mitigate $B_{1}{ }^{+}$Inhomogeneity Using Spatially Selective Radiofrequency Excitation with Generalized Spatial Encoding Magnetic Fields
}

\author{
Yi-Cheng Hsu, ${ }^{1,2}$ I-Liang Chern, ${ }^{1}$ Wei Zhao, ${ }^{3}$ Borjan Gagoski, ${ }^{4}$ Thomas Witzel, ${ }^{3}$ and Fa- \\ Hsuan $\operatorname{Lin}^{2,5 *}$
}

Purpose: High-field magnetic resonance imaging (MRI) has the challenge of inhomogeneous $B_{1}{ }^{+}$, and consequently inhomogeneous flip angle distribution, which causes spatially dependent contrast and makes clinical diagnosis difficult.

Method: We propose a two-step pulse design procedure in which (1) a combination of linear and nonlinear spatial encoding magnetic fields (SEMs) is used to remap the $B_{1}{ }^{+}$map in order to reduce the dimensionality of the problem, (2) the locations, amplitudes, and phases of spoke pulses are estimated in one dimension. The advantage of this $B_{1}{ }^{+}$remapping is that when the isointensity contours of a linear combination of SEMs are similar to the isointensity contours of $B_{1}{ }^{+}$, a simple pulse sequence design using time-varying SEMs can achieve a homogenous flip-angle distribution efficiently.

Results: We demonstrate that spatially selective radiofrequency (RF) excitation with generalized SEMs (SAGS) using both linear and quadratic SEMs in a multi-spoke $k$-space trajectory can mitigate the $B_{1}^{+}$inhomogeneity at $7 \mathrm{~T}$ efficiently. Numerical simulations based on experimental data suggest that, compared with other methods, SAGS provide a formulation allowing multiple-pulse design, a similar average flip-angle distribution with less RF power, and/or a more homogeneous flip-angle distribution.

Conclusion: Without using multiple RF coils for parallel transmission, SAGS can be used to mitigate the $B_{1}{ }^{+}$inhomogeneity in high-field MRI experiments. Magn Reson Med 000:000-000, 2013. ( 2013 Wiley Periodicals, Inc.

Key words: $7 \mathrm{~T}$; RF inhomogeneity; nonlinear gradient; SAR; fast imaging

\footnotetext{
${ }^{1}$ Department of Mathematics, National Taiwan University, Taipei, Taiwan.

${ }^{2}$ Department of Biomedical Engineering and Computational Science, Aalto University School of Science, Espoo, Finland.

${ }^{3}$ A. A. Martinos Center, Department of Radiology, Massachusetts General Hospital, Charlestown, Massachusetts, USA.

${ }^{4}$ Center for Fetal-Neonatal Neuroimaging and Developmental Science, Boston Children's Hospital, Boston, Massachusetts, USA.

${ }^{5}$ Institute of Biomedical Engineering, National Taiwan University, Taipei, Taiwan.

Grant sponsor: National Science Council (NSC), Taiwan; Grant number: 101-2628-B-002-005-MY3, 100-2325-B-002-046. Grant sponsor: Ministry of Economic Affairs, Taiwan; Grant number: 100-EC-17-A-19-S1-175. Grant sponsor: National Health Research Institute, Taiwan; Grant number: NHRIEX102-10247EI. Grant sponsor: Finland Distinguished Professor (FiDiPro) programme.

${ }^{*}$ Correspondence to: Fa-Hsuan Lin, Ph.D., Institute of Biomedical Engineering, National Taiwan University, Taipei, Taiwan. E-mail: fhlin@ntu.edu.tw Received 26 August 2012; revised 26 March 2013; accepted 17 April 2013 DOI 10.1002/mrm.24801

Published online in Wiley Online Library (wileyonlinelibrary.com).
}

(c) 2013 Wiley Periodicals, Inc.

High-field magnetic resonance imaging (MRI) shows a great promise due to its high signal-to-noise ratio $(1,2)$. Yet one challenge of the high-field MRI is the inhomogeneous flip-angle distribution when a volume radiofrequency (RF) coil is used for RF excitation (3). This artifact is due to the deleterious interaction of the dielectric properties of the sample with the shorter wavelength of the RF fields in a strong main magnetic field $B_{0}(>4 \mathrm{~T})$ (4-6). Consequently, an imaging object with the size approximating to a human head can have a spatially varying flip-angle distribution with larger flip angles at the center of the field-of-view (FOV) and smaller flip angles at the periphery of the FOV (2). This causes images with a spatially dependent $T_{1}$ contrast, which is difficult for clinical diagnosis.

Different methods for mitigating $\mathrm{B}_{1}{ }^{+}$inhomogeneity have been proposed. Dedicated volume RF transmit coils have been designed (7-9). Spatially selective RF excitation (10) can be used to design RF and gradient waveforms in order to generate a homogeneous flip-angle distribution after considering the inhomogeneous $B_{1}^{+}$ generated by a volume coil (11). Under the practical limit of relatively short RF excitation (in a few milliseconds), this approach can only mitigate a rather smooth flip-angle distribution below 7T. Using simultaneous RF excitation from multiple RF coils, it has been shown that RF shimming $(12-16)$ and transmit SENSE $(17,18)$ methods can use a shorter RF pulse than the spatially selective RF excitation method to achieve the desired flip-angle distribution. Several parallel RF excitation strategies have been demonstrated at 3T $(19,20)$, 4.7T (20), and $7 \mathrm{~T}(21,22)$. However, the challenges include the complexity of the RF electronics and coils to achieve simultaneous excitation, the necessity of accurate estimates of phases and amplitudes of the $B_{1}{ }^{+}$maps for each RF coil, and the specific absorption rate (SAR) management (23). Alternatively, a more homogeneous image intensity can be obtained after appropriately combining images of different modes of a volume coil (24). However, this approach is designed to improve the image intensity inhomogeneity rather than to reduce the flipangle inhomogeneity.

Recently, it has been demonstrated that nonlinear spatial encoding magnetic fields (SEMs) can be used in MRI spatial encoding in order to improve spatiotemporal resolution $(25,26)$. Preliminary studies using quadratic SEMs for RF excitation (27-32) and small FOV imaging (33) have also been reported. Under the small flip-angle approximation, a formulation to describe the spatial 
distribution of the flip angle when RF pulse is transmitted with time-varying linear and nonlinear SEMs has been described earlier $(28,29,34)$. Furthermore, methods of using linear and quadratic SEMs to compensate $B_{1}{ }^{+}$ inhomogeneity have been proposed. Specifically, parallel RF transmission can be combined with higher spatial frequency encoding at the periphery of the FOV generated by a quadratic SEM to reduce flip-angle inhomogeneity $(35,36)$. Alternatively, driving linear and quadratic SEMs between two excitation pulses can generate spatially dependent transverse magnetization phase, which can counteract the inhomogeneous $B_{1}^{+}$and consequently leads to more homogeneous flip-angle distribution (37).

Here, under the small flip-angle approximation (10), we propose the spatially selective RF excitation using generalized SEMs (SAGS), a two-step pulse design procedure in which (1) a combination of linear and nonlinear SEMs is used to remap the $B_{1}{ }^{+}$map, so as to reduce the dimensionality of the problem, (2) the locations, amplitudes, and phases of spokes pulses are estimated in one dimension. The advantage of this $B_{1}{ }^{+}$remapping is that when the isointensity contours of a linear combination of SEMs are similar to the isointensity contours of $B_{1}{ }^{+}$, a simple pulse sequence design using time-varying SEMs can be used to achieve a homogenous flip-angle distribution efficiently. Using simulations based on empirical data at $7 \mathrm{~T}$, we compare our method with fast- $k_{\mathrm{z}}$ (11) and the method of tailored excitation using nonlinear $B_{0}$-shims (37). Results demonstrate that SAGS can achieve homogeneous flip-angle distribution with a low $\mathrm{RF}$ power without parallel RF transmission.

\section{THEORY}

Small Flip-Angle Approximation Using Nonlinear SEMs With Inhomogeneous $B_{1}^{+}$

For an MRI system with $n$ distinct configurations of SEMs turning on during RF excitation, we use the dimensionless variable $\mathbf{f}(\mathbf{r})=\left[f_{1}(\mathbf{r}), \ldots, \mathrm{f}_{n}(\mathbf{r})\right]$ to describe the spatial distributions of the z-components of these SEMs. To facilitate the description of the arbitrary spatial distribution of $\mathbf{f}(\mathbf{r})$, including nonpolynomial distributions, we define that the maximal and the minimal values among all components of $\mathbf{f}(\mathbf{r})$ within the imaging object are 1 and 0 , respectively. $\mathbf{g}(t)=\left[\mathrm{g}_{1}(t) \ldots \mathrm{g}_{\mathrm{n}}(t)\right]$ describes the instantaneous magnetic field strength of each individual SEM in a physical unit. Accordingly, each component of $\mathbf{g}(t)$ clearly defines the instantaneous difference between the minimal and maximal $z$-component of the magnetic field generated by each SEM within the imaging object. The instantaneous additional $z$-component of the magnetic field at location $\mathbf{r}$ is thus the inner product $\mathbf{g}(t) \cdot \mathbf{f}(\mathbf{r})$. Here, we assume that the $\mathrm{RF}$ transmit field $B_{1}^{+}(\mathbf{r}, t)$ is spatiotemporally separable $B_{1}{ }^{+}(\mathbf{r}, t)=B_{1}{ }^{+}(\mathbf{r}) \times B_{1}{ }^{+}(t)$, where $B_{1}{ }^{+}(t)$ is a waveform of the RF transmit field, and $B_{1}{ }^{+}(\mathbf{r})$ is a spatial distribution of the ratio between $B_{1}{ }^{+}(\mathbf{r}, t)$ and $B_{1}{ }^{+}(t)$.

Taking the small flip-angle approximation and assuming the initial magnetization $\left[M_{x}(\mathbf{r}, 0), M_{y}(\mathbf{r}, 0), M_{z}(\mathbf{r}, 0)\right]^{\mathrm{T}}=$ $[0,0,1]^{\mathrm{T}}$, the spatial distribution of the transverse magnetization $M_{x y}(\mathbf{r})$, RF pulse waveform $B_{1}{ }^{+}(t)$, and temporal integral of SEMs (linear/nonlinear MRI gradients) over time are related to each other by an inverse Fourier transform $(28,29,34)$ :

$$
\begin{aligned}
& M_{x y}(\mathbf{r})=\int_{K} W(\mathbf{k}) B_{1}^{+}(\mathbf{r}) \exp [j 2 \pi \mathbf{f}(\mathbf{r}) \cdot \mathbf{k}] s(\mathbf{k}) d \mathbf{k} \\
& W(\mathbf{k})=W(\mathbf{k}(t))=\frac{j \gamma B_{1}^{+}(t)}{\left|\mathbf{k}^{\prime}(t)\right|} \\
& \mathbf{k}(t)=\left[k_{1}(t), \cdots, k_{n}(t)\right]=\gamma \int_{t}^{T} \mathbf{g}(s) d s
\end{aligned}
$$

Note that the notation $\mathbf{k}(t)$ in this study is different from that in conventional MRI (10). We chose $\mathbf{k}(t)$ to express the maximal phase difference of the transverse magnetization precession within the imaging object at time instant $t . \mathbf{k}(t)$ is the product between the gradient moment and the gyromagnetic ratio $\gamma$. Since a $k$-space trajectory has the one-to-one correspondence between $\mathbf{k}(t)$ and $t$, we omit the $t$ argument in $\mathbf{k}(t)$ and use $\mathbf{k}$ in the following. Additionally, we use a delta function $s(\mathbf{k})$ in $k$-space to describe the $k$-space trajectory.

To achieve a practical slice-selective RF excitation, we propose to use a spoke $k$-space trajectory (11). Without losing generality, we consider that the slice selection is in the $z$-axis and that only the central slice $(z=0)$ is excited. Accordingly, Eq. 1 in a spoke $k$-space trajectory becomes

$$
M_{x y}(x, y)=B_{1}^{+}(x, y) \sum_{\text {spokes }} W_{F}\left(\mathbf{k}_{f}\right) \exp \left[2 \pi j \mathbf{k}_{f} \cdot \mathbf{f}(x, y)\right],
$$

where $M_{x y}(x, y)$ is the excited transverse magnetization at the $z=0$ plane.

\section{The $k$-Space Dimension Reduction Using Nonlinear SEMs}

When linear $X$ and $Y$ SEMs are used, Eq. 2 shows that $W_{\mathrm{F}}\left(\mathbf{k}_{\mathrm{f}}\right)=W_{\mathrm{F}}\left(k_{\mathrm{x}}, k_{\mathrm{y}}\right)$ is the 2D discrete Fourier transform coefficient of $M_{x y}(x, y) / B_{1}{ }^{+}(x, y)$. Suppose that we aim to achieve a target transverse magnetization distribution $M_{x y}(x, y)$ subject to the $B_{1}{ }^{+}$field $B_{1}{ }^{+}(\mathbf{r})=B_{1}{ }^{+}(x, y)$ at $z$ $=0$. If $M_{x y}(x, y) / B_{1}{ }^{+}(x, y)$ can be parameterized by $\mathbf{f}(x, y)$, then Eq. 2 becomes

$$
\begin{aligned}
M_{x y}(x, y) / B_{1}^{+}(x, y) & =\tilde{M}_{x y}(\mathbf{f}(x, y)) \\
& =\sum_{\text {spokes }} W_{F}\left(\mathbf{k}_{f}\right) \exp \left[2 \pi j \mathbf{k}_{f} \cdot \mathbf{f}(x, y)\right]
\end{aligned}
$$

This shows that the $W_{\mathrm{F}}\left(\mathbf{k}_{\mathrm{f}}\right)$ is the discrete Fourier transform coefficient of $\tilde{M}_{x y}(\mathbf{f}(x, y))$. The dimension of this discrete Fourier transform depends on the dimension of $\mathbf{f}(x, y)$. Using generalized SEM can be advantageous if there is an one-dimensional $f(x, y)$ such that

$$
M_{x y}(x, y) / B_{1}^{+}(x, y) \approx \tilde{M}_{x y}(f(x, y)) .
$$

Then, Eq. 3 becomes 


$$
\tilde{M}_{x y}(f(x, y))=\sum_{\text {spokes }} W_{F}\left(k_{f}\right) \exp \left[2 \pi j k_{f} f(x, y)\right] .
$$

$W_{\mathrm{F}}\left(k_{f}\right)$ is the 1D discrete Fourier transform coefficient of $\tilde{M}_{x y}(f(x, y))$. Reducing the dimension of the discrete Fourier transform from 2D to 1D implies that a shorter $k$-space trajectory can be used to achieve the similar distribution of the transverse magnetization.

\section{Design the Flip-Angle Distribution Using Arbitrary SEMs and a Spoke $k$-Space Trajectory}

A target transverse magnetization distribution can be achieved efficiently using arbitrary SEMs and a spoke $k$ space trajectory, if Eq. 4 is satisfied. If targeting on achieving a spatially homogeneous flip-angle distribution $M_{x y}(x, y)=m_{x y}$, this criterion is equivalent to finding one configuration of SEMs such that the isointensity contours of $f(x, y)$ is geometrically similar to the isointensity contours of $B_{1}^{+}(x, y)$. Since in this situation the value of $M_{x y}(x, y) / B_{1}^{+}(x, y)$ can only depend on which isointensity curve of $f(x, y)$ it lies on.

When Eq. 4 is satisfied, the next is to design the location $k_{f}$ and the strength $W_{\mathrm{F}}\left(k_{f}\right)$ as requested by Eq. 5. A $\tilde{M}_{x y}(f(x, y))$ can be generally approximated accurately by a sufficient number of spokes satisfying the Nyquist sampling rate. However, since $\tilde{M}_{x y}(f(x, y))$ is typically spatially smooth, it may be more efficient using irregularly spaced spokes to achieve the approximation of Eq. 5 using fewer spokes. This idea is similar to the fast$k_{z}$ method, which uses spokes near the center of the $k$ space to achieve a quadratic transverse magnetization distribution. In practice, we propose using irregularly spaced spokes, whose strengths $W_{\mathrm{F}}\left(k_{f}\right)$ can be estimated by subjecting to the least-square error criterion (error $=$

$\left.M_{x y}(x, y)-B_{1}(x, y) \sum_{\text {spokes }} W_{F}\left(k_{f}\right) \exp \left[2 \pi j k_{f} \cdot f(x, y)\right]\right) \quad$ and locations can be exhaustively sought over a set of candidate locations.

\section{METHOD}

Comparison on Relative RF Power and Pulse Duration Between SAGS and Fast-k Methods

We used simulations to study if a quadratic flip-angle distribution can be more efficiently achieved by a configuration of SEM using a two-spoke trajectory than the fast- $k_{\mathrm{z}}$ method. The fast $-k_{\mathrm{z}}$ method used five spokes: one central spoke at the center of the $k_{x}-k_{y}$ plane $\left(k_{x}, k_{y}\right)=(0,0)$. Four side spokes were at the $\left(k_{\mathrm{x}}, k_{\mathrm{y}}\right)=(0,0.32),(0$, $-0.32),(0.32,0)$, and $(-0.32,0)$. The ratio of the amplitude between the central spoke and a side spoke was 1:0.175. The locations, amplitudes, and phases of each spoke were chosen manually to generate a quadratic transverse magnetization distribution with the magnitude of the transverse magnetization $\left|M_{x y}\right|=0.25$ at center and $\left|M_{x y}\right|=0.5$ at the corner of the FOV without considering the $\left|B_{1}{ }^{+}\right|$inhomogeneity. This flip-angle distribution can be used to mitigate the $\left|B_{1}{ }^{+}\right|$inhomogeneity, where $\left|B_{1}{ }^{+}\right|$ tends to be higher in the center of the FOV (11).

In this simulation, the SAGS method used the Z2 SEM $\left(f_{\mathrm{Z} 2}(x, y, z) \propto z^{2}-1 / 2\left(x^{2}+y^{2}\right)\right)$ and a two-spoke $k$-space trajectory. Two spokes were at $k_{\mathrm{f}}=0.077$ and $k_{\mathrm{f}}=$ -0.077 with $49.85 \%$ amplitude of the central spoke in the fast- $k_{\mathrm{z}}$ method. The locations, amplitudes, and phases of two spokes were manually tuned to generate a $\left|M_{x y}\right|$ distribution close to the $\left|M_{x y}\right|$ distribution generated by five spokes described above.

The RF power for the fast- $k_{\mathrm{z}}$ method and SAGS was separately calculated as the sum of the squares of the amplitude of each spoke. We also plot the $k$-space trajectories and the corresponding pulse sequences for both methods (Fig. 1). The pulse sequences were calculated based on a $25 \times 25 \times 25 \mathrm{~cm}^{3}$ FOV and a 5 -mm slice using SEMs with a maximal slew rate of $800 \mathrm{~T} / \mathrm{s}$ within the FOV and with the maximal strength generating 160 $\mathrm{mT}$ difference in the $z$-component of the magnetic field within the FOV. This slew rate and maximal strength was equivalent to the $200 \mathrm{~T} / \mathrm{m} / \mathrm{s}$ slew rate and $40 \mathrm{mT} / \mathrm{m}$ maximal strength in classical MRI using linear SEMs. Note that a recent study using the Z2 SEM for spatial encoding used a higher maximal strength than our simulation specification (38).

\section{$\left|B_{1}{ }^{+}\right|$Map of Saline Phantom and Human Head}

The $B 1^{+}$map of a saline phantom was acquired on a 7T system (Siemens, Erlangen, Germany) with a 16-channel receive array, a SC72 gradient (maximum amplitude 70 $\mathrm{mT} / \mathrm{m}$, maximum slew rate $200 \mathrm{~T} / \mathrm{m} / \mathrm{s}$ ), and a 16-channel transmit array. Two healthy subjects with the informed consents were also scanned on the same 7T scanner with a 16-channel transmit array driven by a Butler matrix in the birdcage mode and a 16-channel wrap-around receive coil array. We collected the $B_{1}{ }^{+}$map at the magnet isocenter with $10 \mathrm{~mm}$ thickness, $240 \times 240 \mathrm{~mm}^{2} \mathrm{FOV}$, and $64 \times 64$ image matrix. The $B_{1}^{+}$mapping method consisted of two parts: first, acquiring low flip-angle images and then stepping through different flip angles of the presaturation pulse (39). Imaging parameters were as follows: $\mathrm{TR} / \mathrm{TE}=1000 \mathrm{~ms} / 2 \mathrm{~ms}$.

\section{SAGS Design Procedure to Mitigate $\left|B_{1}{ }^{+}\right|$Inhomogeneity}

\section{Find the SEM With Profile $f(x, y)$ Satisfying Eq. 4}

In this study, we only used the linear and quadratic SEMs. At the $z=0$ plane, $f(x, y)$ is a quadratic function with unknown coefficients. Since we desire a homogeneous flip-angle distribution with a constant $M_{x y}(x, y)=$ $m_{x y}$, the goal is to find $f(x, y)$ and $\tilde{M}(f)$ such that $\tilde{M}_{x y}(f(x, y)) \approx m_{x y}(x, y) / B_{1}^{+}(x, y)$. This equation suggests that we need to find $f(x, y)$ such that the isointensity contour of $f(x, y)$ matches the isointensity contours of $B_{1}{ }^{+}(x, y)$. It is possible to determine $f(x, y)$ based on observation. For example, the isointensity contours of the $B_{1}{ }^{+}(x, y)$ of a saline phantom are nearly concentric ellipses. Thus, we used isocontours of $B_{1}{ }^{+}(x, y)$ to determine the center of the ellipses. Then, we averaged ratios between the long and short axes of each ellipse to estimate the shape of the ellipse.

The isointensity contours of the $B_{1}{ }^{+}(x, y)$ in the human head can be too complicated to be determined by observation. Under the assumption that the $B_{1}{ }^{+}$map is spatially smooth, we first chose to approximate the desired 

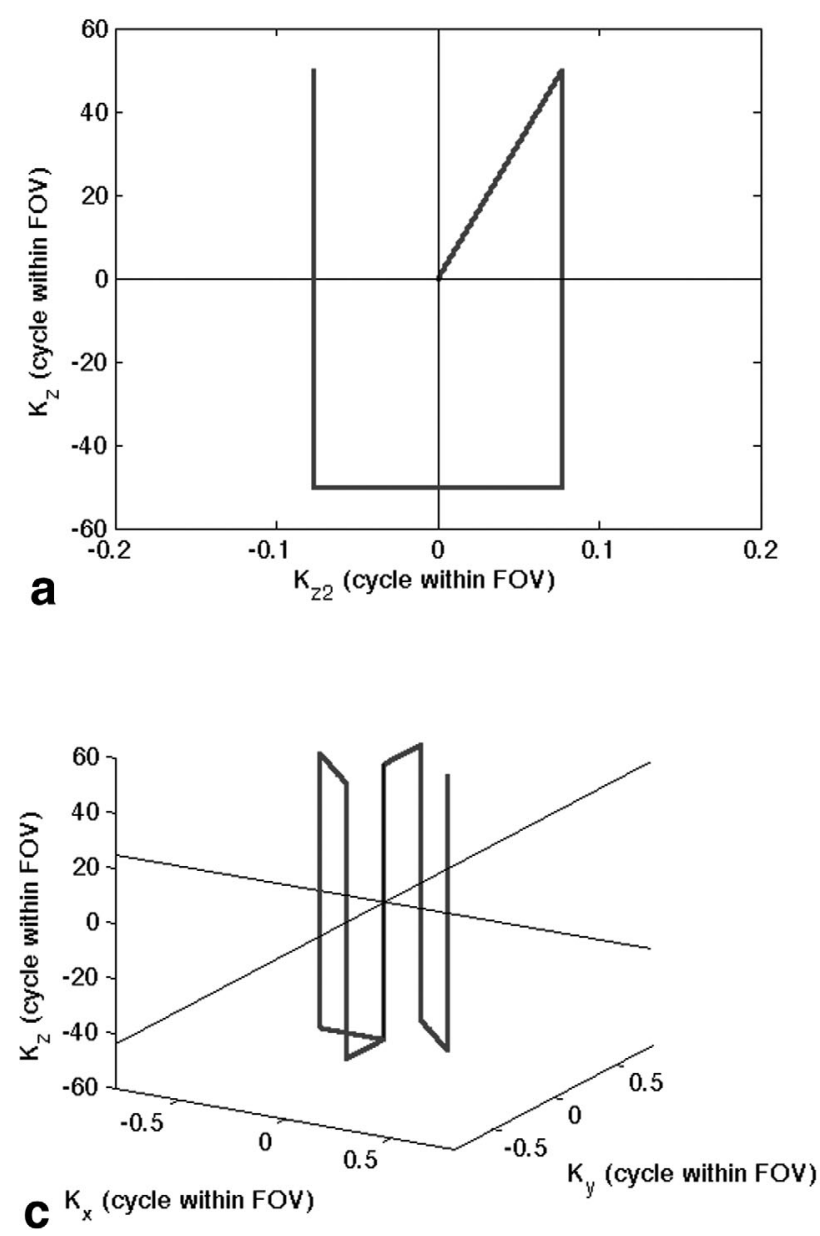

SAGS
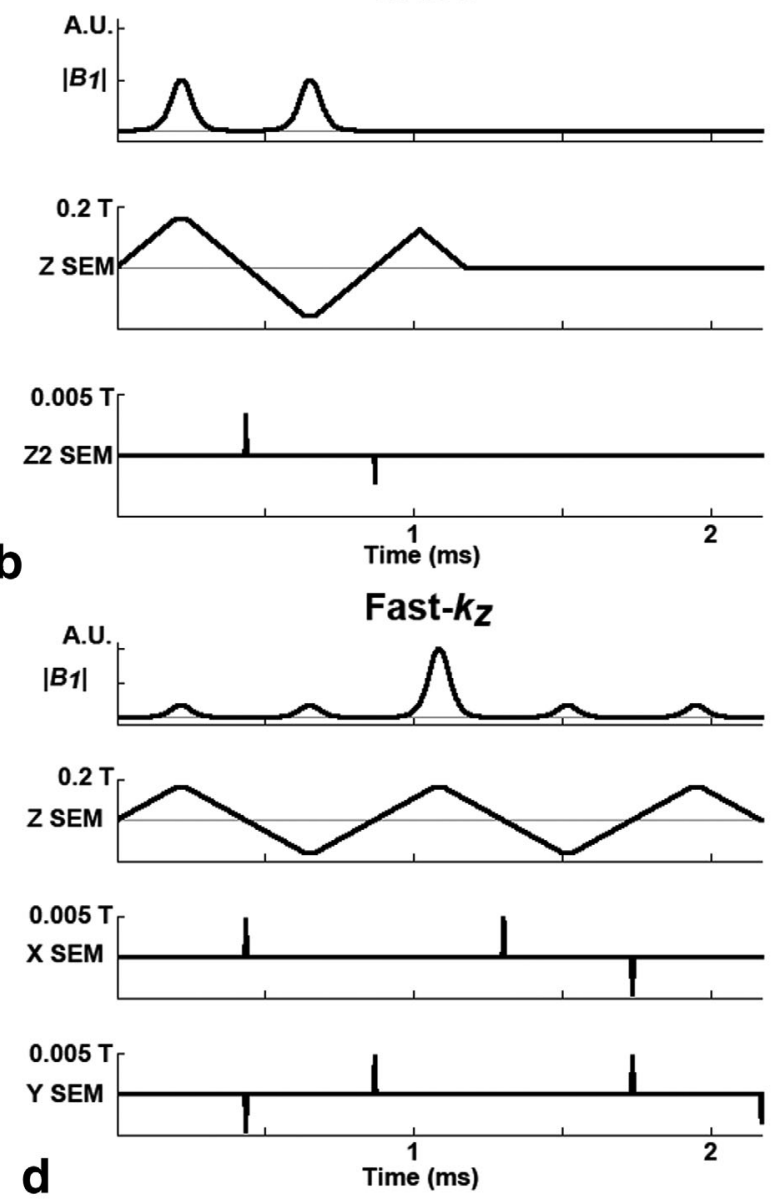

FIG. 1. The $k$-space trajectories $(\mathbf{a}, \mathbf{c})$ and the pulse sequence diagrams $(\mathbf{b}, \mathbf{d})$ of SAGS using two spokes and the fast- $k_{\mathbf{z}}$.

$m_{x y} / B_{1}^{+}(x, y)$ by a distribution consisting of a linear combination of spatial harmonics: $\tilde{M}(f(x, y))=$ $\sum_{l=0}^{L} \alpha_{l} \sin (l f(x, y))+\beta_{l} \cos (l f(x, y))$, where $\alpha_{l}$ and $\beta_{l}$ are the coefficients for the $l$-th harmonic used in the approximation. Realistically $\alpha_{l}$ and $\beta_{l}$ were estimated using a gradient decent method with the initial guess $\tilde{M}_{0}(f(x, y))$ estimated by using only the first harmonic: $\tilde{M}_{0}(f(x, y))=\beta_{1} \cos (f(x, y)) . \quad \beta_{1}$ is the smallest number such that $m_{x y} / \beta_{1}<1$, and $f(x, y)=a x^{2}+b y^{2}+c x y+d x$ $+e y+g$ can be uniquely estimated by the least square fitting of arccos $\left(m_{x y} / \beta_{1} B_{1}^{+}(x, y)\right)$ using a quadratic function

$$
\begin{aligned}
(a, b, c, d, e, g)= & \operatorname{argmin}|| \arccos \left(m_{x y} / \beta_{1} B_{1}^{+}(x, y)\right)-a x^{2} \\
& +b y^{2}+c x y+d x+e y+g \|_{2},
\end{aligned}
$$

Overall, the cost function of this procedure is

$$
\begin{aligned}
& \min _{a, b, c, d, e, g, \alpha_{l}, \beta_{l}} \| B_{1}^{+}(x, y) \sum_{l=1}^{L}\left(\alpha_{l} \sin (I f(x, y))+\beta_{l} \cos (I f(x, y))\right) \\
& \quad-m_{x y} \|_{2}
\end{aligned}
$$

We used the gradient descent method to optimize coefficients in Eq. 7. Afterward, $f(x, y)$ was linearly scaled such that its minimum and the maximum are 0 and 1 within the imaging object, respectively.

To evaluate if Eq. 4 is satisfied, we plot the distribution between $f(x, y)$ and normalized $m_{x y} / B_{1}{ }^{+}(x, y)$, which was linearly scaled $m_{x y} / B_{1}{ }^{+}(x, y)$ such that the maximum $=1$. Ideally, the distribution of all data points should be on a curve, which was estimated by fitting all points using a 10th-order polynomial. We calculated the normalized standard deviation, which was the standard deviation of the difference between the fitted polynomial and each data point divided by the mean of the normalized $m_{x y} / B_{1}^{+}(x, y)$ to quantify the fitting error in Eq. 4 .

Find Spoke Locations and Compute the Amplitudes and Phases for Each Spoke

In the second step, the locations, amplitudes, and phases of spokes were estimated. First, design the location of spokes. Physically, we know that $\tilde{M}_{x y}(f(x, y))$ is a smooth function and nearly monotonically increasing from the center to the periphery of the FOV. Hence, a spoke at $k_{f} \in[-0.5,0.5]$ may be sufficient to approximate this distribution, because a spoke at $k_{f}=+1$ or -1 represents one full cycle oscillation within the imaging 
object. To find the locations of the $n$ spokes, we also assumed that spokes are symmetrically located at $K_{f}=\left\{ \pm k_{i}\right\}$, where $k_{0}<0.5$ and $k_{i}<k_{i-1}+1, i=1 \ldots n-1$.

We then estimated the amplitude and phase of each spoke. For any $K_{f}$, we estimated $W_{f}\left(\left\{ \pm k_{i}\right\}\right)$ by the leastsquares solution of Eq. 2. Since this least-squares solution can be calculated rather fast, we sought all possible $k_{i}$ in steps of 0.05 . We calculated results of two and four spokes in this study.

\section{Comparison on Relative RF Power and $\left|M_{x y}\right|$ Homogeneity Between One-Pulse, SAGS, Fast- $k_{z}$, and Two-Pulse "Tailored-Excitation" Methods}

For comparison, we also estimated the $\left|M_{x y}\right|$ distributions generated by (1) one spoke using only the linear $z$ SEM, (2) a fast- $k_{\mathrm{z}}$ five-spoke trajectory using only linear SEMs (11), and (3) a two-spoke "tailored-excitation" trajectory using both linear $(X$ and $Y$ ) and quadratic ( $Z 2$, X2-Y2, XY) SEMs (37). Note that the two-spoke tailored excitation has a similar $k$-space trajectory as SAGS method. With a predetermined spoke amplitude and constrained by the desired flip-angle distribution, the two-spoke tailored excitation first estimates the phase distribution accrued between two spokes. Given the latency between two spokes, SEMs are then chosen independently to approximate the desired spatially dependent phase shift to approximate this phase distribution, which is subsequently used to compensate the $\left|M_{x y}\right|$ inhomogeneity. Based on the chosen spoke amplitudes a priori, there are either the most homogeneous $\left|M_{\mathrm{xy}}\right|$ (the most homogeneous mode) or the least RF power dissipation (the minimal SAR mode). For the fast- $k_{\mathrm{z}}$ method, the locations of side spokes were symmetrically located on the $\left|k_{\mathrm{x}}\right|<0.5$ and $\left|k_{\mathrm{y}}\right|<0.5$. At each possible spoke location, we estimated spoke amplitude and phase using the least-squares solution of Eq. 2 without using any regularization. The locations of spokes in the fast- $k_{\mathrm{z}}$ trajectory were sought exhaustively at a step of 0.05 . The five spokes with the least-squares error were chosen as the fast- $k_{\mathrm{z}}$ five-spoke trajectory. All $\left|M_{x y}\right|$ distributions were calculated by solving the Bloch equation numerically with the provided trajectory and SEMs. In addition, we chose to achieve $20^{\circ}$ flipangle distribution when we designed parameters for different methods.

The performance of $\left|B_{1}^{+}\right|$mitigation was evaluated by the relative standard deviation $\sigma(37)$ :

$$
\sigma=\operatorname{std}\left(\left|M_{x y}\right|\right) / \operatorname{mean}\left(\left|M_{x y}\right|\right),
$$

where $\operatorname{std}(\bullet)$ and mean $(\bullet)$ denote taking the standard deviation and the mean, respectively. Note that $\sigma$ changes moderately when the target flip angle is small, because the standard deviation and mean of transverse magnetization are linearly proportional to each other. To explore the limit of SAGS and to justify the number of spokes is sufficient, we parametrically increased the number of equally spaced spoke from 1 to 99 and calculated the relative standard deviation $\sigma$ in phantom and head imaging experiments. We also defined the relative RF power $\eta$ :
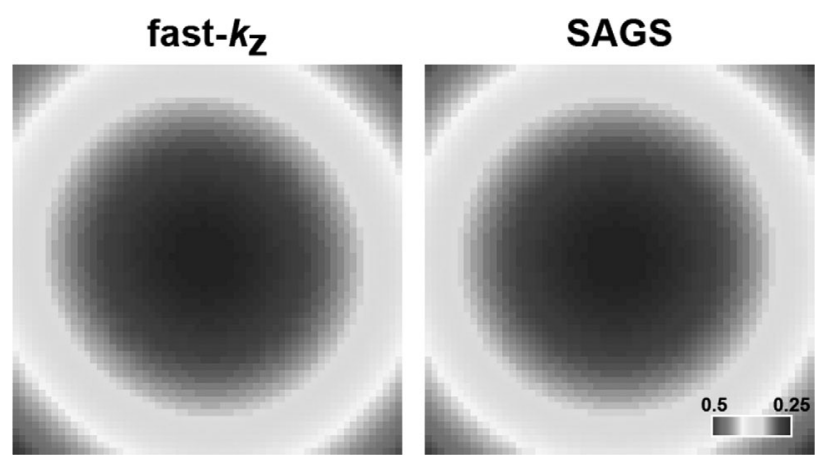

FIG. 2. The distribution of $\left|M_{x y}\right|$ by fast- $k_{z}$ using five spokes and SAGS using two spokes. SAGS used $44 \%$ of the RF power of fast $-k_{\mathrm{z}}$. Results were generated using the pulse sequence and the $k$-space trajectory in Figure 1.

$$
\eta=\sum_{k_{f}}\left|W\left(k_{f}\right)\right|^{2} /\left|W_{\text {single }}\left(k_{f}=0\right)\right|^{2}
$$

where $\left|W_{\text {single }}\left(k_{f}=0\right)\right|$ is the amplitude of the single spoke in the least-square solution to the target transverse magnetization distribution.

\section{RESULTS}

Comparison on Relative RF Power and Pulse Duration Between SAGS and Fast- $k_{z}$ Methods

Figure 1 shows the $k$-space trajectories and the pulse sequence diagrams of SAGS with two spokes and the fast $-k_{\mathrm{z}}$ method. Note that with the simulated SEM, the duration of blips for the Z2 SEM was $9 \mu \mathrm{s}$. The simulated $\left|M_{x y}\right|$ distributions without considering physical $B_{1}{ }^{+}$were shown in Figure 2. Both show similar lower $\left|M_{x y}\right|$ at the FOV center and higher $\left|M_{x y}\right|$ at the FOV periphery. However, compared with the fast- $k_{\mathrm{z}}$ method using five spokes, SAGS used only two spokes to achieve this $\left|M_{X y}\right|$ distribution. Importantly, SAGS used only $44 \%$ of the RF power of fast- $k_{\mathrm{z}}$.

\section{$\left|B_{1}{ }^{+}\right|$Map of Saline Phantom and Human Head}

The estimated $\left|B_{1}^{+}\right|$maps of a saline phantom and two different brain image slices from two different subjects are shown in Figure 3. In the saline phantom, the ratio between the highest and the lowest $\left|B_{1}{ }^{+}\right|$was 3.1. This ratio in head slice 1 and 2 was 2.65 and 3.39, respectively. We found that the $\left|B_{1}{ }^{+}\right|$map of the saline phantom has elliptical isointensity contours. These isointensity contours for the human head $\left|B_{1}{ }^{+}\right|$maps were more irregular.

\section{Mitigate $\left|B_{1}{ }^{+}\right|$Inhomogeneity Using SAGS}

Figure 4 shows the spatial distribution of the $z$ component of the SEM $f(x, y)$ used for $\left|B_{1}{ }^{+}\right|$inhomogeneity mitigation in a saline phantom and two different head imaging slices. Visually, the isointensity contours in Figure 3 are similar to those in Figure 4. The coefficients to generate these SEMs were $(X, Y, Z 2, X 2-Y 2, X Y)=$ $(-0.04,-0.03,-1.98,0.05,0.17),(0.21,-0.22,-2.87$, $1.19,0.13)$, and $(0.14,0.24,-0.16,-0.80,-0.64)$ for 
saline phantom

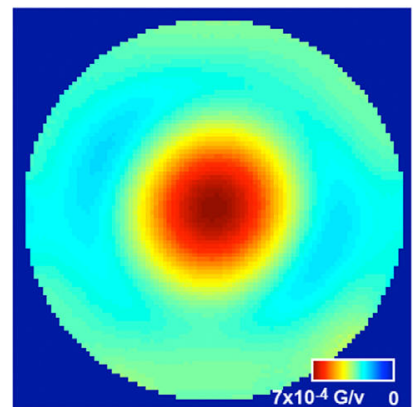

head (slice 1)

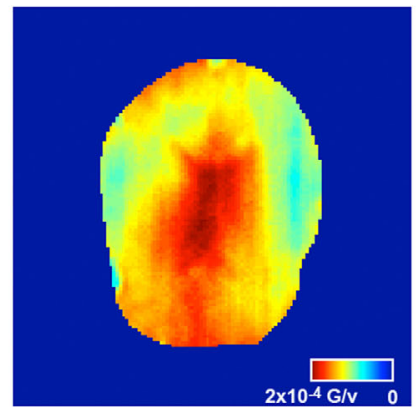

head (slice 2)

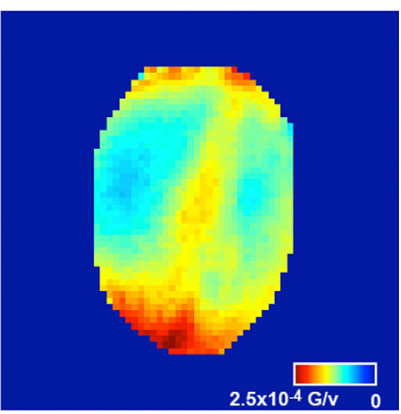

FIG. 3. The estimated $\left|B_{1}{ }^{+}\right|$from a saline phantom and a human head. phantom, head slice 1, and head slice 2, respectively. These coefficients were calculated based on two specifications: (1) FOV ranged between -0.5 and +0.5 , and (2) the minimal and maximal values of the SEM in the imaging object were 0 and 1, respectively. To quantify the similarity, Figure 5 shows the distribution of pairs of $m_{x y} / B_{1}{ }^{+}(x, y)$ and $f(x, y)$ for all image pixels within the imaging object. We found that for the saline phantom, the locations of pairs of $m_{x y} / B_{1}^{+}(x, y)$ and $f(x, y)$ are almost on a curve when $f(x, y)<0.2$. This suggests that the approximation required by Eq. 4 was accurate around the center of the imaging object (region corresponding to $f(x, y)<0.2)$. However, at locations further away from the center of the imaging object, the approximation became poor as the plot showing a scattered distribution. The normalized standard deviation for the saline phantom is 0.0828 . For human head imaging, the approximation required by Eq. 4 was reasonably achieved, since the data points were distributed over a narrow curved band. The normalized standard deviation for the head slice 1 and 2 are 0.1015 and 0.1304 , respectively.

Based on the estimated experimental $\left|B_{1}{ }^{+}\right|$distributions and an arbitrarily chosen $20^{\circ}$ flip angle $\left(\left|M_{\mathrm{xy}}\right|=0.342\right)$, Figure 6 shows simulated $\left|M_{x y}\right|$ in the saline phantom using a single spoke, fast- $k_{\mathrm{z}}$, two-spoke tailored excitation, and SAGS with two or four spokes. The spatial distribution of $\left|M_{x y}\right|$ in single-spoke excitation was similar to the spatial distribution of $\left|B_{1}{ }^{+}\right|$with $\sigma=$ $28.3 \%$ (relative RF power $\eta=1$; Fig. 6a). The two-spoke tailored excitation method with the minimally required RF power can generate the distribution of $\left|M_{X y}\right|$ with $\sigma=$ $21.8 \%$ and $\eta=0.937$ (minimal SAR mode; Fig. 6d) or the most homogeneous distribution of $\left|M_{x y}\right|$ (most homogeneous mode) with $\sigma=7.5 \%$ using a much larger RF power $\left(\eta=19.16\right.$; Fig. 6e). The fast $-k_{\mathrm{z}}$ with five spokes had $\sigma=15.5 \%$ and with $\eta=0.837$ (Fig. 6f). SAGS using two spokes had $\sigma=10.2 \%$ and $\eta=0.64$ (Fig. 6b). SAGS using four spokes had $\sigma=7.4 \%$ and $\eta=$ 0.646 (Fig. 6c). We found that $\sigma$ did not further decrease as the number of spoke increased from 4 to 6 (data not shown). Table 1 lists the spoke amplitudes and phases as well as the associated average and standard deviation of $\left|M_{x y}\right|$.

For the human head slice 1, the spatial distribution of $\left|M_{x y}\right|$ in the single-spoke excitation had $\sigma=18.6 \%(\eta=$ 1; Fig. 7a). The two-spoke tailored excitation method with the minimally required power (minimal SAR mode) can generate the distribution of $\left|M_{x y}\right|$ with $\sigma=9.9 \%$ with $\eta=1.73$ (Fig. 7c) or the "most homogeneous" distribution of $\left|M_{x y}\right|$ with $\sigma=3.9 \%$ using much larger RF power $\eta=34.8$ (most homogeneous mode; Fig. $7 \mathrm{~d}$ ). The fast $-k_{\mathrm{z}}$ with five spokes had $\sigma=11.26 \%$ and with $\eta=$ 1.14 (Fig. 7e). SAGS using two spokes had $\sigma=9.9 \%$ and $\eta=0.92$ (Fig. 7b). SAGS using four spokes had similar $\sigma$ and $\eta$ with that using two spokes (data not shown). Table 2 lists the spoke amplitudes and phases as well as the associated average and standard deviation of $\left|M_{x y}\right|$.

For head slice 2, the spatial distribution of $\left|M_{x y}\right|$ using single-spoke excitation had $\sigma=22.9 \%(\eta=1$; Fig. 8a). The two-spoke tailored excitation method with the minimally required $\mathrm{RF}$ power can generate the distribution of $\left|M_{x y}\right|$ with $\sigma=14.1 \%$ with $\eta=1.61$ (minimal SAR mode; Fig. 8d) or the most homogeneous distribution of $\left|M_{x y}\right|$ with $\sigma=7.7 \%$ using much larger RF power (most homogeneous mode; $\eta=26.9$; Fig. $8 \mathrm{e})$. The fast $-k_{\mathrm{z}}$ method using five spokes had $\sigma=17.0 \%$ and $\eta=0.92$ (Fig. 8f). SAGS using two spokes had $\sigma=13.4 \%$ and $\eta=1.13$

\section{saline phantom}

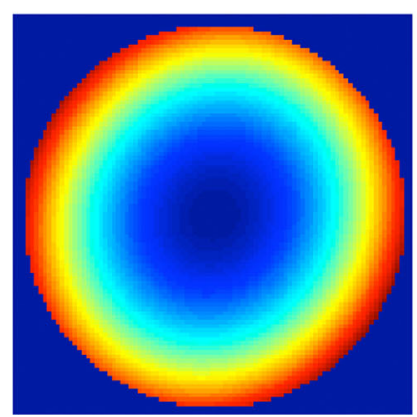

head (slice 1)

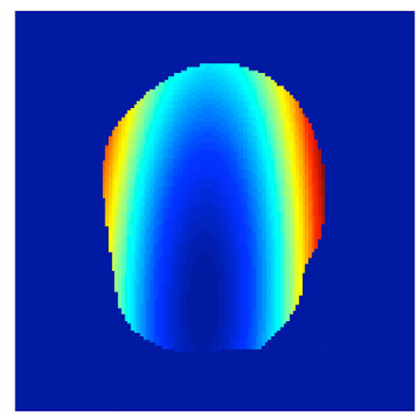

head (slice 2)

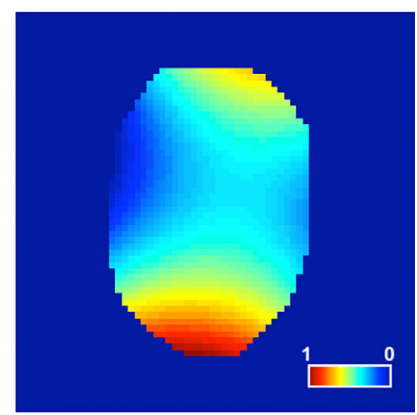

FIG. 4. The spatial distributions of the SEMs used to mitigate the transverse magnetization $M_{x y}$ inhomogeneity in saline phantom (left), human head slice 1 (center), and human head slice 2 (right) experiments. 

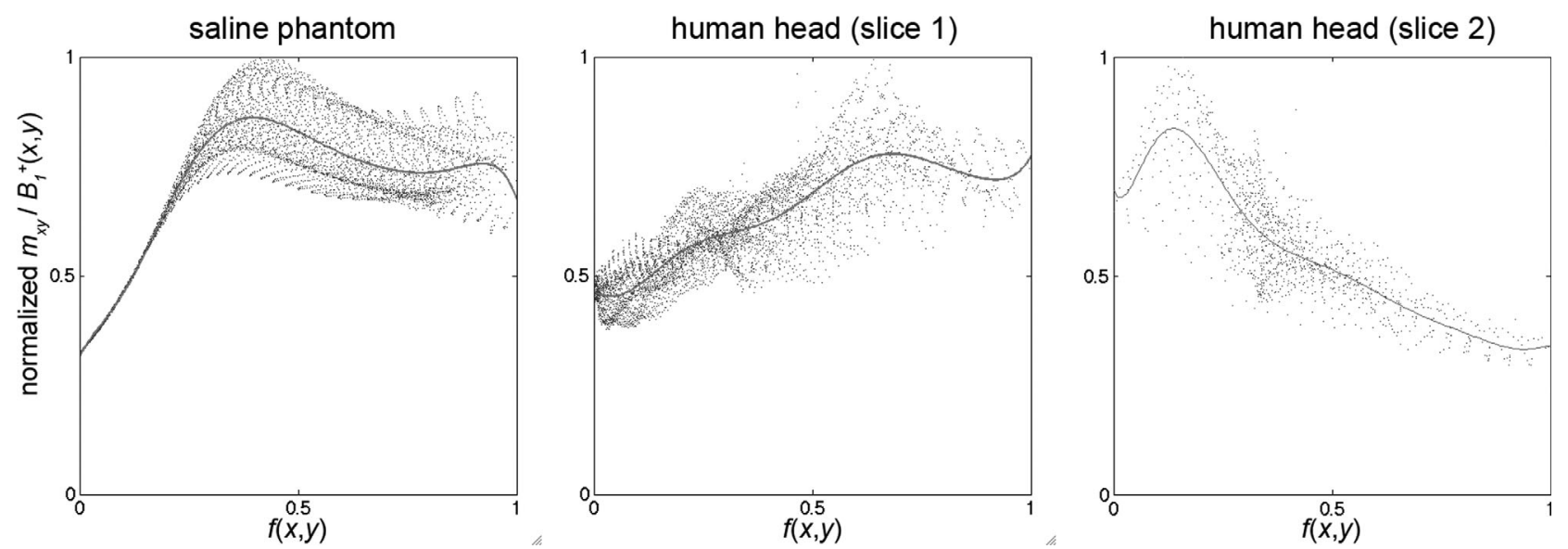

FIG. 5. The $m_{x y} / B_{1}{ }^{+}(x, y)-f(x, y)$ plot for saline phantom (left), the human head slice 1 (center), and the human head slice 2 (right). Blue dots represent the normalized empirical $m_{x y} / B_{1}^{+}(x, y)$ and calculated $f(x, y)$. The red solid curve represents the least-squares error fitting to blue dots using up to the 10th order polynomials.

(Fig. 8b). SAGS using four spokes had $\sigma=12.6 \%$ and $\eta$ $=1.51$ (Fig. 8c). To facilitate the comparison, we plot the profiles of $\left|M_{x y}\right|$ through the center of the image in Figure 9. Table 3 lists the spoke amplitudes and phases as well as the associated average and standard deviation of $\left|M_{x y}\right|$.

Figure 10 shows the relative standard deviation $\sigma$ when the number of equally spaced spokes increased from 1 to 99 in phantom and head imaging experiments. We observe that $\sigma$ decreases very quickly when only nine spokes were used. There was only marginal improvement when more spokes were used. Using 99 spokes had $\sigma=7.3 \%, 9.9 \%$, and $12.5 \%$ for phantom, head slice 1 , and head slice 2 , respectively.

\section{DISCUSSION}

Under the small flip-angle approximation (10), we propose a method to remap the $B_{1}^{+}$map into a lower

FIG. 6. The simulated $\left|M_{x y}\right|$ using measured saline phantom $B_{1}^{+}$ generated by a: one spoke, b: SAGS using two spokes, c: SAGS using four spokes, d: two-spoke tailored excitation with the minimally required power, e: twospoke tailored excitation with the most homogeneous result, and f: the fast- $k_{z}$ method. $\sigma$ is the relative standard deviation of $\left|M_{x y}\right|$ and $\eta$ is the relative RF power. dimension coordinate system. The advantage of this remapping is that when the isointensity contours of either linear or nonlinear SEMs are similar to the isointensity contours of $B_{1}^{+}$, a simple pulse sequence design using time-varying linear and nonlinear SEMs can be used to achieve a homogenous flip-angle distribution efficiently. Specifically, we demonstrate the benefit of using linear and quadratic SEMs to reduce the dimension of the $k$-space (and thus faster and less RF power) to improve flip-angle homogeneity, when the isointensity contours of the chosen SEM are similar to the isointensity contours of the desired transverse magnetization divided by $\left|B_{1}^{+}\right|$. This advantage was demonstrated in simulations using linear $z$ SEM and quadratic SEMs and a two-spoke SAGS trajectory to generate a transverse magnetization distribution similar to that generated by the fast $k_{\mathrm{z}}$ method with only $44 \%$ of the RF power (Fig. 2). Empirical results in saline phantom (Fig. 6) and human head (Figs. 7 and 8) further demonstrate that the

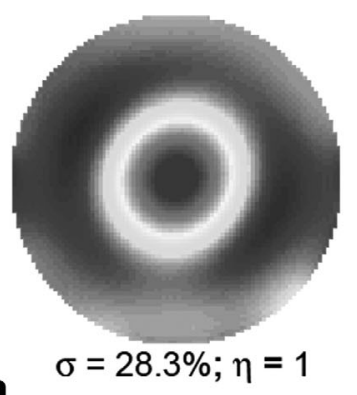

a

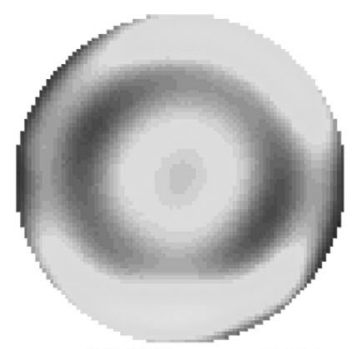

b

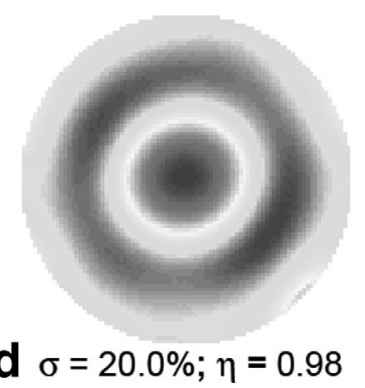

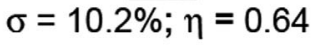

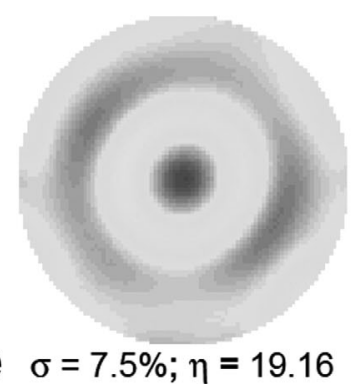

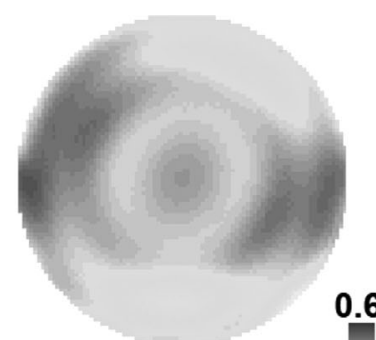

$\sigma=7.4 \% ; \eta=0.65$

C

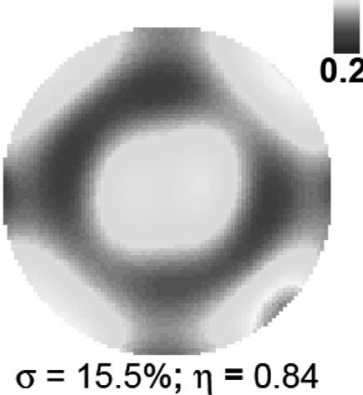


Table 1

Locations, Amplitudes, and Phases of Spokes and the Associated Average and Standard Deviation of the Amplitude of the Transverse Magnetization in the Saline Experiment at $7 \mathrm{~T}$

\begin{tabular}{|c|c|c|c|c|c|}
\hline Method & Spoke & Amplitude (a.u.) & Phase (rad.) & Avg. $\left(\left|M_{x y}\right|\right)$ & Std. $\left(\left|M_{x y}\right|\right)$ \\
\hline Single spoke & 1 & 1 & 0 & 0.3186 & 0.0903 \\
\hline \multirow[t]{5}{*}{ Fast- $k_{z}$} & Central & 0.8956 & 0 & 0.3316 & 0.0514 \\
\hline & Side 1 & 0.1141 & $\pi$ & & \\
\hline & Side 2 & 0.0769 & $\pi$ & & \\
\hline & Side 3 & 0.0769 & $\pi$ & & \\
\hline & Side 4 & 0.1141 & $\pi$ & & \\
\hline \multirow[t]{2}{*}{ Tailored excitation (minimal power) } & 1 & 0.7000 & 0 & 0.3602 & 0.0722 \\
\hline & 2 & 0.7000 & 0 & & \\
\hline \multirow[t]{2}{*}{ Tailored excitation (most homogeneous) } & 1 & 3.0952 & 0 & 0.3439 & 0.0255 \\
\hline & 2 & 3.0952 & 0 & & \\
\hline \multirow[t]{2}{*}{ SAGS (2-spoke) } & 1 & 0.5657 & 1.2293 & 0.3304 & 0.0338 \\
\hline & 2 & 0.5657 & -1.2293 & & \\
\hline \multirow[t]{4}{*}{ SAGS (4-spoke) } & 1 & 0.0816 & 2.6496 & 0.3318 & 0.0245 \\
\hline & 2 & 0.5642 & 1.0441 & & \\
\hline & 3 & 0.5642 & -1.0441 & & \\
\hline & 4 & 0.0816 & -2.6496 & & \\
\hline
\end{tabular}

two-spoke SAGS method can generate more homogeneous flip-angle distribution (compared with single-spoke $k$-space trajectory and fast- $k_{\mathrm{z}}$ method using only linear SEMs) or a similar flip-angle homogeneity with much lower RF power (compared with the two-spoke tailored excitation method).

While our method and fast- $k_{z}$ both analyze the spatial distribution of $M_{x y}$ using the small flip-angle approximation, fast $k_{z}$ only uses the linear SEMs, and SAGS uses both linear and nonlinear ones. Nonlinear SEMs are not commonly available in most MRI scanners. Thus, the applicability of the proposed method may be limited. However, SAGS can achieve a more homogeneous distribution of $\left|M_{x y}\right|$ than the fast- $k_{z}$ method with a shorter pulse duration (Fig. 1) and a lower RF power (Figs. 6 and 7).

The reasons that the tailored excitation method gives less homogeneous flip-angle distribution than the SAGS method in the "minimum SAR" mode (Figs. 6-8) are as follows: (1) in the minimum SAR mode, the RF power has been chosen and it is no longer a free parameter. Thus, the degree of freedom is identical between SAGS and the tailored excitation methods. (2) The tailored excitation method attempts to use both linear and quadratic SEMs to approximate the required distribution of the phase accrued between two RF pulses. Under the small flip-angle approximation (as required to satisfy the minimum SAR mode), this accrued phase distribution is

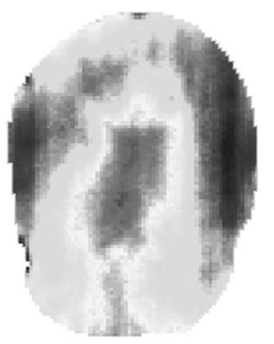

a $\sigma=18.6 \% ; \eta=1$

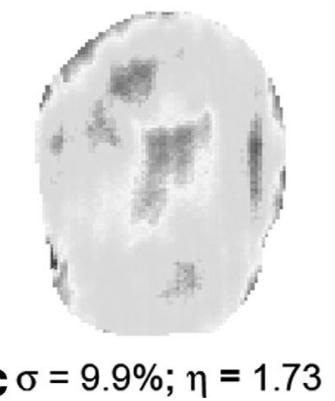

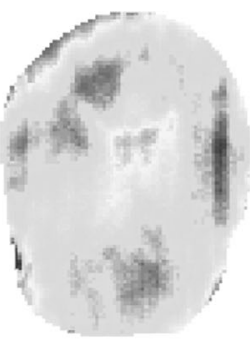

b $\sigma=9.9 \% ; \eta=0.92$

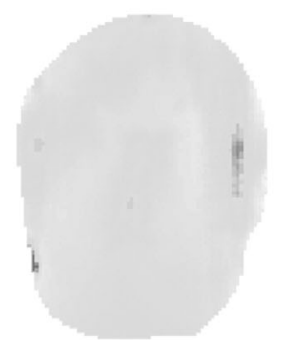

d $\sigma=3.9 \% ; \eta=34.82$
0.5

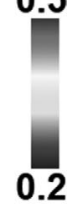

FIG. 7. The simulated $\left|M_{x y}\right|$ using measured human head slice $1 B_{1}^{+}$generated by a: one spoke, b: SAGS using two spokes, c: twospoke tailored excitation with the minimally required power, d: two-spoke tailored excitation with the most homogeneous result, and e: the fast $-k_{z}$ method. $\sigma$ is the relative standard deviation and $\eta$ is the relative RF power. 
Table 2

Locations, Amplitudes, and Phases of Spokes and the Associated Average and Standard Deviation of the Amplitude of the Transverse Magnetization in the Head Slice 1 Experiment at 7T

\begin{tabular}{|c|c|c|c|c|c|}
\hline Method & Spoke & Amplitude (a.u.) & Phase (rad.) & Avg. $\left(\left|M_{x y}\right|\right)$ & Std. $\left(\left|M_{x y}\right|\right)$ \\
\hline Single spoke & 1 & 1 & 0 & 0.3307 & 0.0613 \\
\hline \multirow[t]{5}{*}{ Fast- $k_{z}$} & Central & 1.1606 & 0 & 0.3375 & 0.0393 \\
\hline & Side 1 & 0.1611 & $\pi$ & & \\
\hline & Side 2 & 0.0232 & $\pi$ & & \\
\hline & Side 3 & 0.0232 & $\pi$ & & \\
\hline & Side 4 & 0.1611 & $\pi$ & & \\
\hline \multirow[t]{2}{*}{ Tailored excitation (minimal power) } & 1 & 0.9301 & 0 & 0.3468 & 0.0344 \\
\hline & 2 & 0.9301 & 0 & & \\
\hline \multirow[t]{2}{*}{ Tailored excitation (most homogeneous) } & 1 & 4.1725 & 0 & 0.3425 & 0.0132 \\
\hline & 2 & 4.1725 & 0 & & \\
\hline \multirow[t]{2}{*}{ SAGS (2-spoke) } & 1 & 0.6782 & 0.9759 & 0.3303 & 0.0327 \\
\hline & 2 & 0.6782 & -0.9759 & & \\
\hline
\end{tabular}

$\arccos \left(m_{x y} / \beta B_{1}^{+}(x, y)\right)$. However, it is important to note that the homogeneous distribution of $M_{x y}(\mathrm{x}, \mathrm{y})=m_{\mathrm{xy}}$ is what we aim to achieve ultimately, rather than the distribution of the accrued phase $\arccos \left(m_{x y} / \beta B_{1}^{+}(x, y)\right)$. The nonlinear arccos function and the denominator term $B_{1}{ }^{+}(x, y)$ add undesired weightings on $M_{x y}(\mathrm{x}, \mathrm{y})$. In other words, the spatial distribution of the accrued phase is optimized with the least-square error, but not $M_{x y}(\mathrm{x}, \mathrm{y})$. This may explain why there was only marginal improvement in the absolute value of transverse magnetization homogeneity by the two-spoke tailored excitation using the similar RF power (Fig. 6).

One limit of the SAGS is that the two-spoke tailored excitation method can correctly describe the dynamics of magnetization excited by two high-amplitude spokes, while SAGS needs to use the small flip-angle approximation. Thus, the two-spoke tailored excitation method may achieve better results in cases, where a higher RF power is used to excite magnetization. This is demonstrated in the case, where a higher $\mathrm{RF}$ power can improve the $\left|M_{x y}\right|$ homogeneity (Figs. 6d, 6e, 7c, 7d, 8d and $8 \mathrm{e}$ ). One challenge of the two-spoke tailored

FIG. 8. The simulated $\left|M_{x y}\right|$ using measured human head slice $2 B_{1}{ }^{+}$ generated by a: one spoke, b: SAGS using two spokes, c: SAGS using four spokes, d: two-spoke tailored excitation with the minimally required power, e: two-spoke tailored excitation with the most homogeneous result, and $f$ : the fast $-k_{z}$ method. $\sigma$ is the relative standard deviation and $\eta$ is the relative RF power.

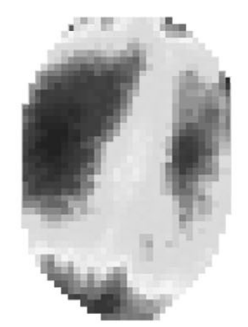

a $\sigma=22.9 \% ; \eta=1$

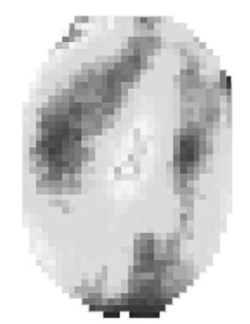

$\mathbf{d} \sigma=14.1 \% ; \eta=1.61$ excitation method is that it is difficult to further generalize to design SEMs when there are more than two pulses. By contrast, our method did not have this limitation.

To our knowledge, there is no method of identifying the globally optimized $k$-space coordinates in $n$-dimension, where $n$ is the number of SEMs. In some cases, it might be easy to identify the appropriate combination of the SEMs such that its isointesnity contours were similar to those of the $B_{1}{ }^{+}$map (like the saline phantom experiment; Fig. 3). There are other cases that the $B_{1}{ }^{+}$map is rather complicated and therefore identifying the combination of the SEMs becomes tedious. If such a combination is identified, optimizing spoke locations can become rather straightforward. In fact, directly fitting SEMs to the target excitation pattern in Step 1 as described in this study is just one of many potential approaches to registering their isocontours; different methods may result in solutions that require fewer spokes than the one described here.

SAGS differs from the method proposed by Grissom et al. (35) by (1) they used parallel RF excitation, but our method uses only one single RF transmitter, and (2) our

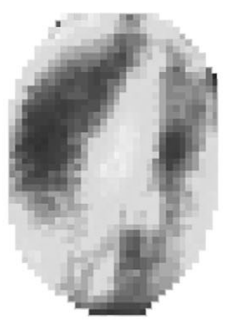

b $\sigma=13.4 \% ; \eta=1.13$

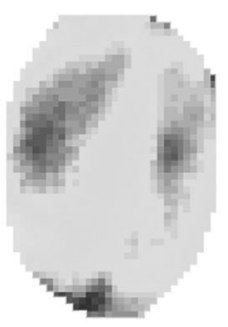

e $\sigma=7.7 \% ; \eta=26.9$

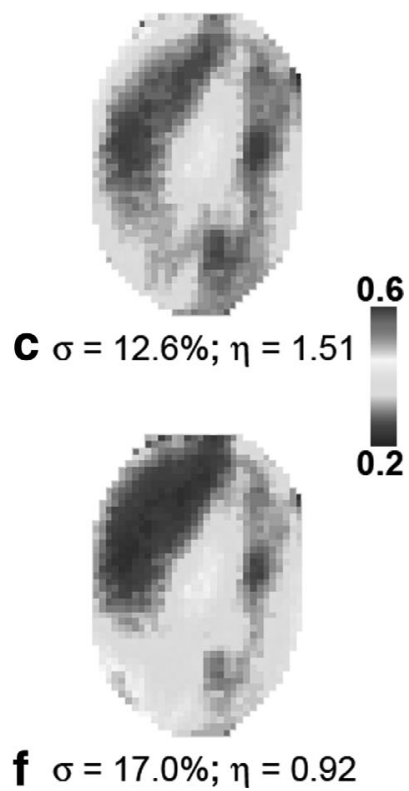



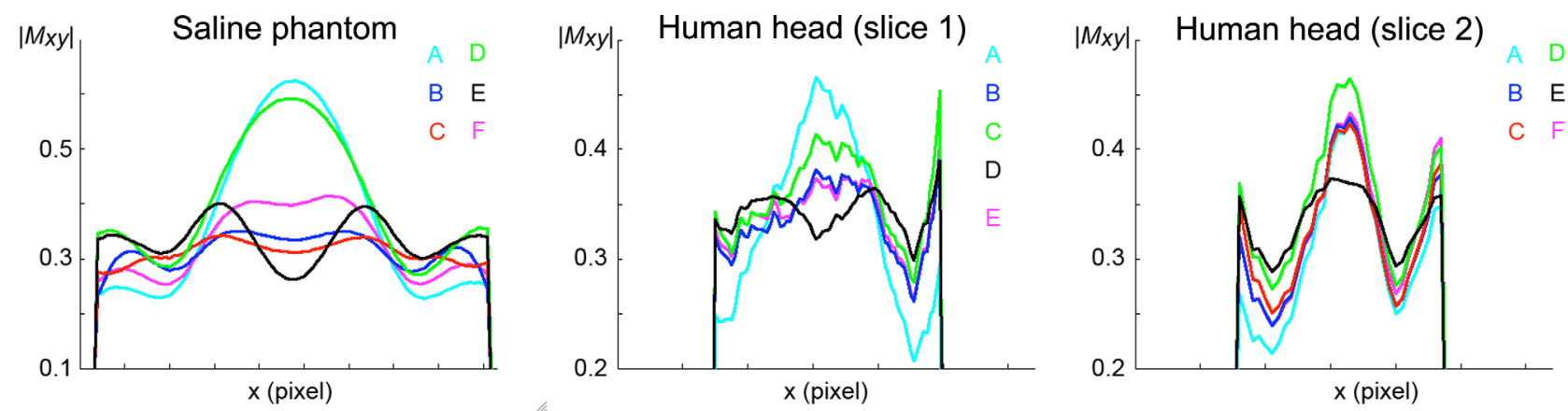

FIG. 9. Left: the horizontal profiles of the simulated $\left|M_{x y}\right|$ at the center of the image using measured saline phantom $B_{1}{ }^{+}$generated by $\mathbf{A}$ : one spoke, B: SAGS using two spokes, C: SAGS using four spokes, D: two-spoke tailored excitation with the minimally required power, E: two-spoke tailored excitation with the most homogeneous result, and F: the fast- $k_{z}$ method. Center: the horizontal profiles of the simulated $\left|M_{x y}\right|$ at the center of the image using measured human head slice $1 \mathrm{~B}_{1}^{+}$generated by A: one spoke, B: SAGS using two spokes, C: twospoke tailored excitation with the minimally required power, D: two-spoke tailored excitation with the most homogeneous result, and $\mathrm{E}$ : the fast $-k_{z}$ method. Right: the horizontal profiles of the simulated $\left|M_{x y}\right|$ at the center of the image using measured human head slice $2 B_{1}{ }^{+}$generated by A: one spoke, B: SAGS using two spokes, C: SAGS using four spokes, D: two-spoke tailored excitation with the minimally required power, E: two-spoke tailored excitation with the most homogeneous result, and $\mathrm{F}$ : the fast- $k_{z}$ method.

method provides a clear description of designing $\mathrm{RF}$ pulses, including $k$-space dimension reduction (i.e., remapping for $B_{1}{ }^{+}$maps) and optimizing the location of RF spokes.

While aiming at achieving a homogeneous flip-angle distribution, SAGS requires no multiple RF amplifiers and $\mathrm{RF}$ coils as required by the parallel transmit $(17,18,21,40)$ and RF shimming techniques (12-16). However, SAGS require quadratic SEMs. Although quadratic SEMs are not commonly available in MRI scanner, it has been demonstrated that the requirement of quadratic SEMs is not high: even quadratic shimming coils can generate very satisfying results (37). Our calculations were based on the second-order nonlinear gradients, whose spatial magnetic field distributions were similar to those of the realistic second-order shimming coils in the most modern MRI, Patloc imaging $(28,29)$, and Ospace imaging (36). Additionally, while parallel transmit $(17,18,21,40)$ and RF shimming techniques (12-16) require accurate $B_{1}{ }^{+}$maps for each RF coils, SAGS only require one accurate $\left|B_{1}^{+}\right|$map.

SAGS has two limitations: first, remapping $B_{1}{ }^{+}$into the coordinates defined by the selected SEM can be imperfect. The second limitation is the number of spokes to approximate the desired $M_{\mathrm{xy}}$ distribution. The first limitation was studied in Figure 5, where we show the error of this remapping. It shows that the more accurately we can remap $B_{1}{ }^{+}$, the more homogeneous flip angle we can achieve. The second limitation was studied in Figure 10, where we parametrically increased the number of the spoke from 1 to 99 . We found that using only a few spokes can achieve a similar result as using 99 spokes.

In spoke pulse design, the orthogonal matching pursuit method can efficiently determine suitable spokes location on a multidimensional $k$-space (41). Typically, the orthogonal matching pursuit method only checks possible spoke locations separated by integer multiples of the

Table 3

Locations, Amplitudes, and Phases of Spokes and the Associated Average and Standard Deviation of the Amplitude of the Transverse Magnetization in the Head Slice 2 Experiment at $7 \mathrm{~T}$

\begin{tabular}{|c|c|c|c|c|c|}
\hline Method & Spoke & Amplitude (a.u.) & Phase (rad.) & Avg. $\left(\left|M_{x y}\right|\right)$ & Std. $\left(\left|M_{x y}\right|\right)$ \\
\hline Single spoke & 1 & 1 & 0 & 0.3516 & 0.0806 \\
\hline \multirow[t]{5}{*}{ Fast $-k_{z}$} & Central & 0.8896 & 0 & 0.3279 & 0.0557 \\
\hline & Side 1 & 0.1405 & $\pi$ & & \\
\hline & Side 2 & 0.2112 & $\pi$ & & \\
\hline & Side 3 & 0.2112 & $\pi$ & & \\
\hline & Side 4 & 0.1405 & $\pi$ & & \\
\hline \multirow[t]{2}{*}{ Tailored excitation (minimal power) } & 1 & 0.8967 & 0 & 0.3492 & 0.0493 \\
\hline & 2 & 0.8967 & 0 & & \\
\hline \multirow[t]{2}{*}{ Tailored excitation (most homogeneous) } & 1 & 3.6684 & 0 & 0.3442 & 0.0264 \\
\hline & 2 & 3.6684 & 0 & & \\
\hline \multirow[t]{2}{*}{ SAGS (2-spoke) } & 1 & 0.7608 & 0.5972 & 0.3259 & 0.0437 \\
\hline & 2 & 0.7608 & -0.5972 & & \\
\hline \multirow[t]{4}{*}{ SAGS (4-spoke) } & 1 & 0.1420 & 0.5298 & 0.3249 & 0.0408 \\
\hline & 2 & 0.8565 & 1.7102 & & \\
\hline & 3 & 0.8565 & -1.7102 & & \\
\hline & 4 & 0.1420 & -0.5298 & & \\
\hline
\end{tabular}




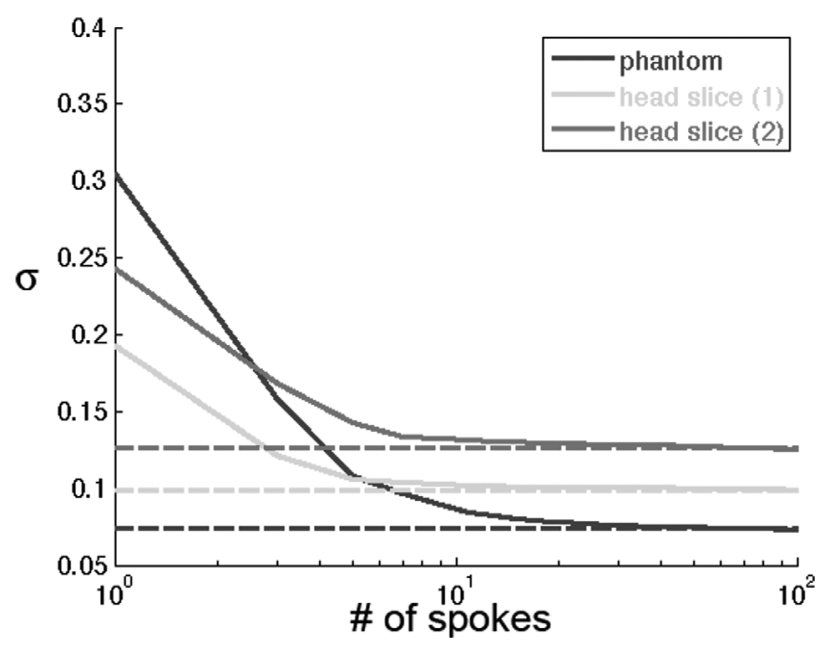

FIG. 10. The normalized standard deviation $\sigma$ of $\left|M_{x y}\right|$ using SAGS with 1 to 99 equally spaced spokes. The dashed line shows the $\sigma$ generated by the SAGS method with two or four spokes (Figs. 6-8).

$k$-space distance determined by the inverse of the FOV and the Nyquist sampling theorem. Grissom et al. (36) generalizes this method by a local optimization method such that the spoke location around these integer modes can also be the candidate of the optimized solution. The motivation of using all integer modes as candidate spoke locations is to approximate any arbitrary $M_{x y}$ distribution by providing complete bases. However, when targeting at reducing $M_{x y}$ inhomogeneity with spatially smooth variation, searching spoke locations around lower-order modes should generate satisfactory results. In fact, exhaustive searching around lower-order mode may generate more efficient excitation. This is demonstrated in our study of using 99 equally spaced spokes (Fig. 10): SAGS with as few as two or four spokes can generate a transverse magnetization distribution similar to the transverse magnetization distribution generated by 99 equally spaced spokes.

The spoke locations, amplitudes, and phases may be estimated by taking the Fourier transform of the 1D target excitation pattern as a function of $f$. However, there are two concerns. Using the Fourier transform to find spoke locations/amplitudes/phases implies that (1) all data points in the remapped domain $f$ are equally important and (2) the optimized spoke locations are separated by integer multiples of $1 / \mathrm{FOV}$. However, the data points in the remapped domain $f$ are not uniformly distributed (Fig. 5). Additionally, spokes separate by integer multiples of $1 / F O V$ does not guarantee the most efficient solution (Fig. 10).

While we only demonstrated SAGS at the central $(z=$ 0) slice, it is possible to use SAGS to excite other slice by shifting the frequency of the excitation pulse to excite a different slice and shifting the center of Z2 gradient by adding a $z$ SEM.

We only used the Gaussian RF pulse to achieve slice selection in the $z$ direction in our study. It is possible to use other waveforms to achieve the similar slice selection. While it may be possible to simultaneously drive Z and other quadratic SEMs to achieve the desired spatial distribution of $\left|M_{x y}\right|$, our study only demonstrates how to design multiple-spoke excitations to drive the linear $\mathrm{Z}$ and quadratic SEMs sequentially. Exploration of driving multiple SEMs simultaneously is certainly interesting yet it is outside the scope of this work.

At high-field MRI, the SAR management becomes an important issue as the electromagnetic wave during $R F$ excitation can cause deleterious interaction to endanger the subject (23). This concern is especially critical in parallel transmission, where RF pulses from multiple coils can potentially combine constructively to elevate local electric fields and eddy currents (23). For SAGS, the SAR management may be qualitatively evaluated by comparing with the SAR of traditional slice-selective excitation using the $\eta$ metric in this study. This is because that under the assumption that the strength of the electric field inside the object is linearly proportional to the square root of the transmitted RF power and that the conductivity does not change with the transmitted RF power, the local SAR defined as the product of conductivity and the square of electric field is proportional to the transmitted power. Importantly, because SAGS does not use multiple coils for parallel RF transmission, the potential RF power hazard from the interaction between RF coils can be avoided.

In conclusion, we proposed the SAGS as a method of design spoke RF pulses using linear and nonlinear SEMs with potential advantage of approximating the desired flip-angle distribution in a lower dimension $k$-space. Without using multiple RF coils for parallel transmission, SAGS can be one method to mitigate the $B_{1}{ }^{+}$inhomogeneity in high-field MRI experiments.

\section{ACKNOWLEGMENT}

The authors thank Dr. Lawrence L Wald for his help on the $7 \mathrm{~T}$ data.

\section{REFERENCES}

1. Hoult D, Richards R. The signal to noise ratio of the nuclear magnetic resonance experiment. J Magn Reson 1976;24:71-85.

2. Vaughan JT, Garwood M, Collins CM, et al. 7T vs. 4T: RF power, homogeneity, and signal-to-noise comparison in head images. Magn Reson Med 2001;46:24-30.

3. Bottomley PA, Andrew ER. RF magnetic field penetration, phase shift and power dissipation in biological tissue: implications for NMR imaging. Phys Med Biol 1978;23:630-643.

4. Collins CM, Liu W, Schreiber W, Yang QX, Smith MB. Central brightening due to constructive interference with, without, and despite dielectric resonance. J Magn Reson Imag 2005;21:192-196.

5. Van de Moortele PF, Akgun C, Adriany G, Moeller S, Ritter J, Collins CM, Smith MB, Vaughan JT, Ugurbil K. B(1) destructive interferences and spatial phase patterns at $7 \mathrm{~T}$ with a head transceiver array coil. Magn Reson Med 2005;54:1503-1518.

6. Vaughan JT, Garwood M, Collins CM, et al. 7T vs. 4T: RF power, homogeneity, and signal-to-noise comparison in head images. Magn Reson Med 2001;46:24-30.

7. Alsop DC, Connick TJ, Mizsei G. A spiral volume coil for improved RF field homogeneity at high static magnetic field strength. Magn Reson Med 1998;40:49-54.

8. Vaughan JT, Adriany G, Snyder CJ, Tian J, Thiel T, Bolinger L, Liu H, DelaBarre L, Ugurbil K. Efficient high-frequency body coil for highfield MRI. Magn Reson Med 2004;52:851-859.

9. Adriany G, Van de Moortele PF, Ritter J, Moeller S, Auerbach EJ, Akgun C, Snyder CJ, Vaughan T, Ugurbil K. A geometrically adjustable 16-channel transmit/receive transmission line array for improved 
RF efficiency and parallel imaging performance at 7 Tesla. Magn Reson Med 2008;59:590-597.

10. Pauly JM, Nishimura DG, Macovski A. A k-space analysis of smalltip-angle excitation. J Magn Reson 1989;81:43-56.

11. Saekho S, Yip CY, Noll DC, Boada FE, Stenger VA. Fast-kz threedimensional tailored radiofrequency pulse for reduced B1 inhomogeneity. Magn Reson Med 2006;55:719-724.

12. Ibrahim TS, Lee R, Baertlein BA, Abduljalil AM, Zhu H, Robitaille PM. Effect of RF coil excitation on field inhomogeneity at ultra high fields: a field optimized TEM resonator. Magn Reson Imag 2001;19:1339-1347.

13. Mao W, Smith MB, Collins CM. Exploring the limits of RF shimming for high-field MRI of the human head. Magn Reson Med 2006;56:918-922

14. Collins CM, Liu W, Swift BJ, Smith MB. Combination of optimized transmit arrays and some receive array reconstruction methods can yield homogeneous images at very high frequencies. Magn Reson Med 2005;54:1327-1332.

15. Vaughan T, DelaBarre L, Snyder C, et al. 9.4T human MRI: preliminary results. Magn Reson Med 2006;56:1274-1282.

16. Metzger GJ, Snyder C, Akgun C, Vaughan T, Ugurbil K, Van de Moortele PF. Local B1+ shimming for prostate imaging with transceiver arrays at $7 \mathrm{~T}$ based on subject-dependent transmit phase measurements. Magn Reson Med 2008;59:396-409.

17. Katscher U, Bornert P, Leussler C, van den Brink JS. Transmit SENSE. Magnet Reson Med 2003;49:144-150.

18. Grissom W, Yip CY, Zhang Z, Stenger VA, Fessler JA, Noll DC. Spatial domain method for the design of RF pulses in multicoil parallel excitation. Magn Reson Med 2006;56:620-629.

19. Setsompop K, Wald LL, Alagappan V, Gagoski B, Hebrank F, Fontius U, Schmitt F, Adalsteinsson E. Parallel RF transmission with eight channels at 3 Tesla. Magn Reson Med 2006;56:1163-1171.

20. Ullmann P, Junge S, Wick M, Seifert F, Ruhm W, Hennig J. Experimental analysis of parallel excitation using dedicated coil setups and simultaneous RF transmission on multiple channels. Magn Reson Med 2005;54:994-1001.

21. Setsompop K, Alagappan V, Zelinski AC, Potthast A, Fontius U, Hebrank F, Schmitt F, Wald LL, Adalsteinsson E. High-flip-angle slice-selective parallel RF transmission with 8 channels at $7 \mathrm{~T}$. J Magn Reson 2008;195:76-84.

22. Zelinski AC, Wald LL, Setsompop K, Alagappan V, Gagoski BA, Goyal VK, Adalsteinsson E. Fast slice-selective radio-frequency excitation pulses for mitigating $\mathrm{B}_{1}{ }^{+}$inhomogeneity in the human brain at 7 Tesla. Magn Reson Med 2008;59:1355-1364.

23. Lattanzi R, Sodickson DK, Grant AK, Zhu Y. Electrodynamic constraints on homogeneity and radiofrequency power deposition in multiple coil excitations. Magn Reson Med 2009;61:315-334.

24. Orzada S, Maderwald S, Poser BA, Bitz AK, Quick HH, Ladd ME. RF excitation using time interleaved acquisition of modes (TIAMO) to address B1 inhomogeneity in high-field MRI. Magn Reson Med 2010;64:327-333.

25. Stockmann JP, Ciris PA, Galiana G, Tam L, Constable RT. O-space imaging: Highly efficient parallel imaging using second-order nonlinear fields as encoding gradients with no phase encoding. Magn Reson Med 2010;64:447-456.

26. Hennig J, Welz AM, Schultz G, Korvink J, Liu Z, Speck O, Zaitsev M. Parallel imaging in non-bijective, curvilinear magnetic field gradients: a concept study. MAGMA 2008;21:5-14.
27. Weber H, Gallichan D, Schultz G, Witschey WR, Welz AM, Cocosco CA, Hennig J, M. Z. ExLoc: Excitation and Encoding of Curved Slices. In Proceedings of the 19th Annual Meeting of ISMRM, Montreal, Canada, 2011. p. 2806.

28. Haas M, Ullmann P, Schneider JT, Ruhm W, Hennig J, Zaitsev M. Large tip angle parallel excitation using nonlinear non-bijective Patloc encoding fields. In Proceedings of the 18th Annual Meeting of ISMRM, Stockholm, Sweden, 2010. p. 4929.

29. Schneider JT, Haas M, Ohrel S, Lehr H, Ruhm W, Post H, Hennig J, Ullmann P. Parallel spatially selective excitation using nonlinear non-bijective Patloc encoding fields: experimental realization and first results. In Proceedings of the 19th Annual Meeting of ISMRM, Montreal, Canada, 2011. p. 211.

30. Ma C, Xu D, King KF, Liang ZP. Reduced field-of-view excitation using second-order gradients and spatial-spectral radiofrequency pulses. Magn Reson Med 2013;69:503-508.

31. Kopanoglu E, Yilmaz U, Gokhalk Y, Atalar E. Specific absorption rate reduction using nonlinear gradient fields. Magn Reson Med 2012 doi: $10.1002 / \mathrm{mrm} .24478$

32. Haas M, Ullmann P, Schneider JT, Post H, Ruhm W, Hennig J, Zaitsev M. PexLoc-Parallel excitation using local encoding magnetic fields with nonlinear and nonbijective spatial profiles. Magn Reson Med 2012 doi:10.1002/mrm.24559.

33. Witschey WR, Cocosco CA, Gallichan D, Schultz G, Weber H, Welz AM, Hennig J, M. Z. Localization by nonlinear phase preparation and K-space trajectory design (GradLoc). In Proceedings of the 19th Annual Meeting of ISMRM, Montreal, Canada, 2011. p. 2805.

34. Schneider JT, Haas M, Ruhm W, Hennig J, Ullmann P. Robust spatially selective excitation using radiofrequency pulses adapted to the effective spatially encoding magnetic fields. Magnet Reson Med 2011;65:409-421.

35. Grissom WA, Sacolick L, Vogel MW. $\mathrm{B}_{1}{ }^{+}$inhomogeneity compensation using 3D parallel excitation is enhanced by simultaneous linear and nonlinear gradient encoding. In Proceedings of the 19th Annual Meeting of ISMRM, Montreal, Canada, 2011. 2898.

36. Grissom WA, Khalighi MM, Sacolick LI, Rutt BK, Vogel MW. Smalltip-angle spokes pulse design using interleaved greedy and local optimization methods. Magn Reson Med 2012;68:1553-1562.

37. Duan Q, van Gelderen P, Duyn J. Tailored excitation using nonlinear B0-shims. Magn Reson Med 2012;67:601-608.

38. Stockmann JP, Galiana G, Tam L, Juchem C, Nixon TW, Constable RT. In vivo O-Space imaging with a dedicated $12 \mathrm{~cm} \mathrm{Z2}$ insert coil on a human 3T scanner using phase map calibration. Magn Reson Med 2013;69:444-455.

39. Lee J, Gagoski B, Gumbrecht R, Fautz H-P, Wald LL, Adalsteinsson E. Fast $\mathrm{B}_{1}{ }^{+}$mapping with validation for parallel transmit system in $7 \mathrm{~T}$. In Proceedings of the 18th Annual Meeting of ISMRM, Stockholm, Sweden, 2010. p.2835

40. Zelinski AC, Wald LL, Setsompop K, Alagappan V, Gagoski BA, Goyal VK, Adalsteinsson E. Fast slice-selective radio-frequency excitation pulses for mitigating $\mathrm{B}+1$ inhomogeneity in the human brain at 7 Tesla. Magn Reson Med 2008;59:1355-1364.

41. Chen D, Bornemann F, Vogel MW, Sacolick L, Kudielka G, Zhu Y. Sparse parallel transmit pulse design using orthogonal matching pursuit method. In Proceedings of the 17th Annual Meeting of ISMRM, Honolulu, Hawaii, USA, 2009. p. 171. 